\title{
INSTITUIÇÕES CIENTÍFICAS E TECNOLÓGICAS NO BRASIL
}

\author{
Luana da Silva Ribeiro ${ }^{1}$ \\ Universidade Estadual Paulista (UNESP) \\ luanasribeiro78@gmail.com
}

Hugo Márcio Vieira de Almeida Andrade ${ }^{2}$ Universidade Estadual Paulista (UNESP)

hugomarciovieira@gmail.com

Faíque Ribeiro Lima ${ }^{3}$

Universidade Estadual Paulista (UNESP)

faique.ribeiro@hotmail.com

\begin{abstract}
Resumo
Com o intuito de gerar desenvolvimento, os Estados investem em formas de incentivar a criação de inovações, construindo Sistema Nacional de Inovação em que se articuladam as estruturas de geração de inovações. Pretende-se, por meio desta pesquisa mostrar o quanto as interações entre Estado, Universidade e Empresa colaboram para o ambiente inovativo. Para tal, utilizou-se de revisão bibliográfica em autores que discutem os temas e conceitos afins, e bases de dados do governo. Em países em desenvolvimento como o Brasil, o Estado assume um papel importante para o fomento das inovações. Com o intuito de articular o processo inovativo, investindo em inovação e potencializando a capacidade tecnológica. Porém o Brasil ainda possui um sistema de inovação não consolidado, dependente de tecnologia estrangeira, com uma infraestrutura de CT\&I precária, necessitando de políticas de fomento que aumente a conexão com o sistema produtivo e a incorporação do progresso técnico. Adianta-se que o Brasil tem investido em instituições científicas e tecnológicas, mas ainda há necessidade de desburocratizar, planejar e consolidar a política institucional para que se obtenha melhores resultados.
\end{abstract}

Palavras-chave: Instituições tecnológicas, Inovação, ICT, NIT.

\section{SCIENTIFIC AND TECHNOLOGICAL INSTITUTIONS IN BRAZIL}

\begin{abstract}
In order to generate development, the states invest in ways to encourage the creation of innovations, building a National Innovation System in which structures for the generation of innovations are articulated. It is intended, through this research, to show how much the interactions between State University and Company collaborate for the innovative environment. For that, a bibliographical review was used in authors who discuss the themes and related concepts, and government databases. In developing countries such as Brazil, the State plays an important

\footnotetext{
${ }^{1}$ Possui graduação em Ciências Econômicas pela Universidade Estadual de Maringá (2016). Atualmente é mestranda em Economia pela Universidade Estadual Paulista (UNESP/FCLAR), bolsista e participante do Grupo de Estudos em Economia Industrial (GEEIN). Tem experiência na área de desenvolvimento econômico, com ênfase em Políticas Públicas, Economia industrial e de inovação.

2 Mestrando em Economia pela Universidade Estadual Paulista Júlio de Mesquita Filho. Bacharel em Ciências Econômicas pela Universidade Estadual do Sudoeste da Bahia (2017). Representante discente do Conselho de Pósgraduação em Economia da Unesp, estagiário docente da disciplina de Política e Planejamento Econômico, e Assistente de Pesquisa II do Instituto de Pesquisa Econômica Aplicada. Tem experiência na área de Economia, atuando principalmente nos seguintes temas: Desenvolvimento Social e Econômico, Dívida Pública, Economia Brasileira, Economia Política, Economia Solidária, Educação Tutorial, Inovação e Tecnologias Sociais.

${ }^{3}$ Mestrando em Economia pela Faculdade de Ciências e Letras - UNESP/FCLAr e pesquisador bolsista pela Coordenação de Aperfeiçoamento de Pessoal de Nível Superior - CAPES. Bacharel em Ciências Econômicas pela Universidade Estadual do Sudoeste da Bahia - UESB. Trabalhou na Companhia de Bebidas das Américas - AMBEV. Foi pesquisador bolsista no Programa de Educação Tutorial em Ciências Econômicas da UESB. Tem experiência na organização de eventos acadêmicos e elaboração de artigos científicos, especialmente na área de políticas públicas de desenvolvimento industrial, tecnológico e inovações.
}

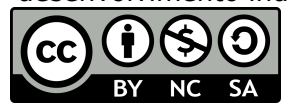

P2P \& INOVAÇÃO, Rio de Janeiro, v. 5 n. 2, p.108-132, Mar./Ago. 2019. 
role in fostering innovation, in order to articulate the innovative process, investing in innovation and enhancing technological capacity. However, Brazil still has a system of unconfined innovation, dependent on foreign technology, with a precarious CT \& I infrastructure, necessitating development policies that increase the connection with the productive system and the incorporation of technical progress. It is anticipated that Brazil has invested in scientific and technological institutions, but there is still a need to reduce bureaucracy, plan and consolidate the institutional policy in order to obtain better results.

Key Words: Technological institutions, Innovation, ICT, NIT.

\section{INTRODUÇÃO}

Na busca de desenvolvimento econômico e social de uma nação, o processo inovativo tem um papel fundamental, por criar capacidades produtivas e, consequentemente, uma maior competitividade internacional. Em países em desenvolvimento como o Brasil, a participação do Estado assume um papel fundamental no fomento da inovação. Uma forma de fomento é o Estado articular a troca de conhecimento entre empresas, universidades e instituições de pesquisa, montando um Sistema Nacional de Inovação capaz de dar condições para a realização de catching-up e a criação de inovações.

A dinâmica do sistema capitalista é um dos fundamentos essenciais para analisar sistemas de inovação, pela razão de que, o ambiente inovativo pode reduzir riscos e incertezas. A interação de redes de pesquisas entre empresas, universidades e instituições de pesquisa funciona como uma possibilidade de minimizar tais riscos e incertezas (AVELLAR; OLIVEIRA, 2008). Segundo a OECD (1997), compreender o Sistema Nacional de Inovação é fundamental por auxiliar na identificação de inadequações no sistema, tanto entre instituições quanto em relação a políticas governamentais, e assim, estimular o desenvolvimento tecnológico e a inovação.

Isto posto, o objetivo do artigo consiste em mostrar o quanto a interação entre Estado, Universidade e Empresa colaboram para o ambiente inovativo. Para que essa interação seja transformada em inovação, é primordial que a base institucional seja consolidada visando o acúmulo de aprendizado e fluxos de conhecimento. Diante disso, será analisado ao final deste artigo as instituições tecnológicas no Brasil.

Em face do objetivo proposto, o artigo está organizado além da introdução e conclusão em três seções. A primeira seção aborda o Sistema Nacional de Inovação e suas tipologias, triângulo de Sábato e a importância das políticas públicas. Na segunda seção desenvolve a interação da universidade-empresa e a terceira seção apresenta-se a relevância e dados das instituições cientificas e tecnológicas no Brasil. 
ARTIGO

\section{INOVAÇÃO}

- SISTEMA NACIONAL DE INOVAÇÃO (SNI): ESTADO, UNIVERSIDADE E INSTITUIÇÕES.

A inovação é entendida como resultado do processo de aprendizado. O processo de aprendizado gera competências e capacitações que são instruídas pela interação entre agentes econômicos e instituições, e assim, a transmissão do conhecimento (correspondente ao processo de aprendizado), passa a ter papel primordial na determinação do desenvolvimento econômico, por solucionar problemas, impulsionar o catching-up e impactar na dinâmica do Sistema Nacional de Inovação (SNI). A inovação é primordial para a sobrevivência das empresas capitalistas, elevação da produtividade, competição entre as firmas, estruturação do comércio internacional entre outros elementos (NELSON; WINTER, 1982; CASSIOLATO; LASTRES, 2000; GIELFI, 2017).

Como abordado no parágrafo anterior, a inovação constante e o conhecimento são essenciais na conjuntura econômica por serem os principais elementos para competitividade e o desenvolvimento econômico das nações. Os países precisam traçar e estruturar um ambiente que seja favorável para o crescimento econômico, consolidando os SNI. Neste âmbito, "a discussão sobre a transferência de conhecimento entre universidade e o setor produtivo não pode ser de forma dissociada da compreensão do conceito de inovação e do sistema inovativo" (FREEMAN; SOETE, 2008; CALDERAN; OLIVEIRA, 2013).

Neste sentido, a ideia de Sistema de Inovação (SI) expandiu-se no início dos anos 90 com os trabalhos comparativos de SNI e estudo da estrutura de um SI nas abordagens de Chris Freeman (1987) e Richard Nelson (1993). Os sistemas de inovação são considerados como uma evolução do conhecimento por intermédio de processos de aprendizagem e inovação.

Um "sistema" define-se como um conjunto de vários elementos relacionados que unidos se transformam em apenas um. Já a esfera "nacional" estipula que todas as partes precisam estar envolvidas no processo e possuir o mesmo ambiente e normas em comum. $\mathrm{O}$ SI pode ser conceituado ainda como os fatores políticos, econômicos, institucionais, sociais, organizacionais, entre outros que são encarregados de gerar, difundir, absorver, modificar conhecimentos e/ou inovações (LUNDVALL, 2004; STRACHMAN; DEUS, 2005; SBICCA E PELAEZ 2006).

O SNI é considerado de uma forma ampla por Lundvall (1992b) como estrutura institucional e econômica que influencia o aprendizado, busca por novos conhecimentos, e que engloba subsistemas dos SNIs (financeiro, marketing, entre outros). De maneira específica, é uma construção institucional dos setores públicos e privados com intuito de 
impulsionar o progresso tecnológico em economias capitalistas complexas por meio da prática de interações que sustentam a transferência de conhecimento visando a geração, modificação e difusão de novas tecnologias (FREEMAN, 1987; ALBUQUERQUE, 1998; FRENKEL, 2001.

Sbicca-Fernandes (2004, p. 2) considera a inovação fundamental no crescimento e produtividade, e os sistemas de inovação, como um processo sistêmico primordial por reunirem diversas instituições ao longo do tempo. Na abordagem de Cassiolato e Szapiro (2015, p. 287), a conceitualização de sistemas de inovação define-se como sendo o desempenho inovativo dependente de um "fenômeno sistêmico e interativo, caracterizado por diferentes tipos de cooperação".

Albuquerque (1998, p. 157) afirma que os arranjos institucionais têm o envolvimento de "redes de interação entre empresas, agências governamentais, universidades, institutos de pesquisa, laboratórios de empresas e atividades de cientistas e engenheiros." Além disso, os arranjos institucionais associam-se com o sistema educacional, industrial, empresarial e instituições financeiras. Deste modo, completa-se "o circuito dos agentes que são responsáveis pela geração, implementação e difusão das inovações".

Neste âmbito, os arranjos institucionais têm a função de tornar endógeno o progresso tecnológico à dinâmica econômica de um país. Portanto, o progresso tecnológico não é proveniente apenas da execução das forças de mercado, mas também do resultado de inovações realizadas pela interação Universidade-Empresa, institutos de pesquisa, órgãos de fomento entre outros (ALBUQUERQUE, 1998; FRENKEL， 2001; CASSIOLATO; SZAPIRO, 2015).

As empresas se conectam em rede umas às outras, apoiando-se em termos de tecnologias e conhecimentos, operando em alta flexibilidade e baixo custo, e promovendo a acumulação de habilidades e a aprendizagem coletiva em benefício de todos os participantes (LUNDVALL, 1992, p. 10).

A interação entre Estado, Universidade e Empresa colabora com o avanço técnico e é considerada integrante do SNIs. Destaca-se também a importância das firmas, centros de pesquisa e o desenvolvimento e aprimoramento de processos e produtos. Nelson e Rosemberg (1993) apontam a magnitude da atuação das universidades, laboratórios de pesquisa, instituições financeiras e o sistema político em geral na composição dos SNI.

More generally, it is somewhat artificial to try to describe and analyze a nation's innovation system as something separable from its economic system more broadly defined, or to depict the policies concerned with innovation as 
quite apart from those concerned with the economy, education, or national security. (NELSON; ROSENBERG, 1993, p. 13).

A dinâmica do sistema capitalista é um dos fundamentos essenciais para analisar sistemas de inovação, pela razão de que, o ambiente inovativo pode reduzir riscos e incertezas. A interação de redes de pesquisas entre empresas, universidades e instituições de pesquisa funciona como uma possibilidade de minimizar tais riscos e incertezas (AVELLAR; OLIVEIRA, 2008).

Para OECD (1997), compreender o SNI é fundamental por auxiliar na identificação de inadequações no sistema, tanto entre instituições quanto em relação a políticas governamentais, e assim, aumentar a eficiência e estimular o desenvolvimento tecnológico e a inovação.

No quadro 1 aborda-se as principais definições de Sistemas Nacionais de Inovação. Percebe-se que a maioria dos autores considera a intervenção do Estado primordial, implementando políticas que favoreçam a interação e transferência de conhecimento entre instituições públicas e privadas no processo de inovação. A interação é relevante por difundir e modificar tecnologias, e por determinar o desempenho inovador.

Quadro 1: Definições de Sistema Nacional de Inovação (SNI)

\begin{tabular}{|c|l|}
\hline $\begin{array}{c}\text { Freeman } \\
\mathbf{( 1 9 8 7 )}\end{array}$ & $\begin{array}{l}\text { A rede de instituições no setor público e privado a qual atividades e } \\
\text { interações desencadeiam importação, modificação e difusão de novas } \\
\text { tecnologias. }\end{array}$ \\
\hline $\begin{array}{c}\text { Lundvall } \\
\mathbf{( 1 9 9 2 )}\end{array}$ & $\begin{array}{l}\text { Os elementos e relações que interagem na produção, difusão e uso } \\
\text { economicamente útil e novo conhecimento que estão localizados } \\
\text { dentro ou enraizados dentro das fronteiras de um estado-nação. }\end{array}$ \\
\hline Nelson (1993) & $\begin{array}{l}\text { Um conjunto de instituições cujas relações determinam o desempenho } \\
\text { inovativo das firmas nacionais. }\end{array}$ \\
\hline $\begin{array}{c}\text { Patel and } \\
\text { Pavitt (1994) }\end{array}$ & $\begin{array}{l}\text { As instituições nacionais, suas estruturas de incentivo e competências, } \\
\text { que determinam a taxa e direção da aprendizagem tecnológica (ou o } \\
\text { volume e a composição atividades que geram mudanças) em um país. }\end{array}$ \\
\hline $\begin{array}{c}\text { Metcalfe } \\
\text { (1995) }\end{array}$ & $\begin{array}{l}\text { O conjunto de instituições diversas que em conjunto e individualmente } \\
\text { contribuem para o desenvolvimento e difusão de novas tecnologias e } \\
\text { fornecem a estrutura no quais governos formulam e implementam } \\
\text { políticas que influenciam o processo de inovação. Assim, é um sistema } \\
\text { de instituições interconectadas para criar, armazenar e transferir os } \\
\text { conhecimentos, habilidades e artefatos que definem novas tecnologias. }\end{array}$ \\
\hline Albuquerque \\
(1996)
\end{tabular} \begin{tabular}{l}
$\begin{array}{l}\text { Uma construção institucional, produto de uma ação planejada e } \\
\text { consciente ou de um somatório de decisões não planejadas e } \\
\text { desarticuladas que impulsiona o progresso tecnológico em economias } \\
\text { capitalistas complexas. }\end{array}$ \\
\hline
\end{tabular}




\begin{tabular}{|c|l|}
\hline $\begin{array}{c}\text { Edquist } \\
(\mathbf{2 0 0 1})\end{array}$ & $\begin{array}{l}\text { É composto por todas entidades econômicas, organizações sociais e } \\
\text { políticas, além de outros fatores que incentivam a inovação e o } \\
\text { desenvolvimento. }\end{array}$ \\
\hline $\begin{array}{c}\text { Sbicca e } \\
\text { Pelaez (2006) }\end{array}$ & $\begin{array}{l}\text { Mesma definição de Freeman (1987), mas, incluem atores dentro do } \\
\text { SNI: Universidades, Estado e Empresas. A interação entre esses atores } \\
\text { é primordial no processo dinâmico e de inovação. }\end{array}$ \\
\hline $\begin{array}{c}\text { Fagerberg e } \\
\text { Srholec } \\
(\mathbf{2 0 0 7 )}\end{array}$ & $\begin{array}{l}\text { Um conjunto de "capacidades sociais" visando o desenvolvimento de } \\
\text { um país. Essas capacidades seriam: competência técnica e de gestão, } \\
\text { governo estável e efetivo e instituições interligadas. }\end{array}$ \\
\hline $\begin{array}{c}\text { Jung e Mah } \\
(\mathbf{2 0 1 3 )}\end{array}$ & $\begin{array}{l}\text { Conjunto de instituições interligadas que tem como objetivo a criação e } \\
\text { exploração de inovações tecnológicas. }\end{array}$ \\
\hline
\end{tabular}

Fonte: Elaboração própria. ALBUQUERQUE, 1996; EDQUIST, 2001; SBICCA; PELAEZ, 2006; FAGERBERG; SRHOLEC, 2007; JUNG; MAH, 2013; OECD, 1997. Tradução nossa.

Nesta conjuntura, o Estado se enquadraria como o responsável no estímulo da capacitação tecnológica, geração da infraestrutura e aplicação de diretrizes para que a política de C\&T promova o desenvolvimento nacional. Ainda neste sentido, o Estado tem a responsabilidade de intervir para impulsionar o desenvolvimento produtivo, tecnológico e a expansão de setores estratégicos.

Em suma, percebe-se que o grau e interação entre diversos agentes que compõe um SNI, é o que determina a capacidade do país na geração de inovação. Mas, para facilitação desta interação é necessário além do Estado, políticas de financiamento e CT\&I. Como aponta nas diversas abordagens da literatura um SNI é complexo e dinâmico, deve organizar-se de forma a proporcionar a articulação dos agentes com intuito de promover inovação em um país (ALBUQUERQUE, 1996; ALBUQUERQUE; SICSÚ, 2000; CASSIOLATO; LASTRES, 2005; SBICCA-FERNANDES; PELAEZ, 2006).

\section{- Tipos de Sistemas de Inovação}

O desenvolvimento tecnológico é classificado como um dos principais motores determinantes da competitividade e das estratégias de desenvolvimento dos países. Há uma forte correlação do grau de desenvolvimento de um país e seu esforço em ciência, tecnologia e inovação. Por isto, é necessário analisar as especificidades. O SNI é classificado em três tipologias: a) sistemas de inovação maduros, são os de países que se enquadram em preservar a nação na fronteira tecnológica; b) sistemas de difusão de inovações, são aqueles de países que investem em tecnologia própria e os que possuem a capacidade de absorver tecnologias dos centros mais avançados; c) sistemas de inovação não consolidados, são os de países que 
dependem da tecnologia estrangeira, cujos SNI não se completaram, ou seja, são imaturos (ALBUQUERQUE, 1996; AVELLAR; OLIVEIRA, 2009).

O Brasil se encaixa na última tipologia por ser um país de industrialização tardia. A infraestrutura mínima de ciência e tecnologia, baixa conexão com o sistema produtivo, debilidade de políticas públicas de fomento e condicionamento de incorporação do progresso tecnológico, foram alguns dos fatores que contribuiu pouco com o desempenho econômico do país (ALBUQUERQUE; SICSÚ, 2000; SUZIGAN; ALBUQUERQUE, 2008).

O SNI brasileiro para Albuquerque $(1998$, p. 160) possui pouca contribuição visando à eficiência no desempenho econômico do país. Podendo-se afirmar que não houve nenhum estágio mínimo que pudesse ser considerado um SNI. Neste contexto, "ao invés de se constituir em uma fonte de "oportunidades tecnológicas como nos sistemas maduros, na periferia a infra-estrutura científica contribui para identificar corretamente oportunidades geradas internacionalmente". Nesta conjuntura, para resolução do problema brasileiro é fundamental a implantação de uma política que vise o desenvolvimento, sendo iniciado nas especificidades locais, além do fomento e criação de novas técnicas (FURTADO, 1961).

É necessário que o Brasil transforme seu SNI integrando os sistemas ou as diferentes instituições envolvidas, além disso, é preciso oferecer apoio para sustentar a estrutura produtiva. Em Cassiolato e Lastres (2005, p. 26) “Cabe ao Estado o caráter pró-ativo voltado para coordenação e indução dos processos de transformação produtiva, visando internalizar os benefícios potenciais proporcionais por tecnologias de um novo paradigma tecnológico".

\subsection{Triângulo de Sábato}

Neste sentido, desenvolvido por Sábato e Botana (1968), o Triângulo de Sábato busca entender o processo de desenvolvimento das economias latino-americanas através da relevância da interação de três dimensões: universidades (infraestrutura científicotecnológica), setor produtivo e governo. Gielfi (2017, p. 36) “A ideia é que estas três dimensões constituem os vértices do triângulo, sendo cada um deles composto por um conjunto de atores e instituições específicas entre si - a chamada intrarrelação ou relação intravértice." Além disso, o triângulo também possui relações entre os vértices, chamado de inter-relação.

Figura 1 - Triângulo de Sábato 


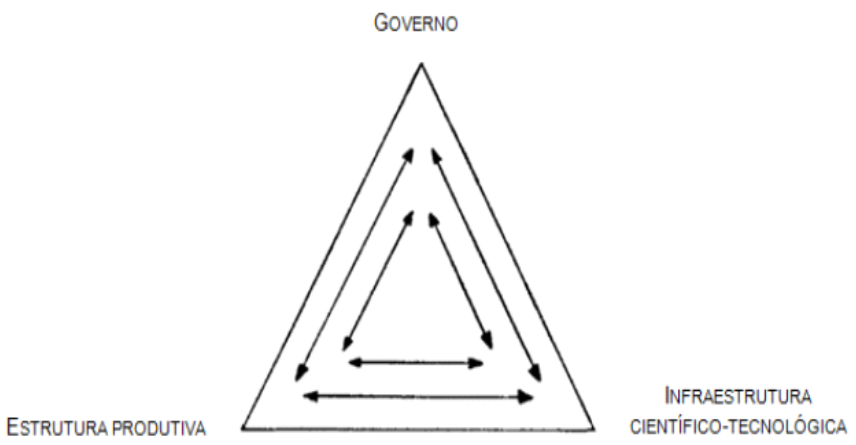

Fonte: Adaptado de SÁBATO; BOTANA, 1968, p. 27.

No topo do triângulo está a vértice governo, e por intermédio da inter-relação se encontra os outros dois vértices que estabelece ações para o desenvolvimento científico e tecnológico e o setor produtivo. Neste sentido, o governo é o principal agente que opera sobre os vértices, planejando políticas e ações, executando e conduzindo a infraestrutura de C\&T e a estrutura produtiva (inter-relações horizontais do triângulo).

Gielfi (2017, p. 36) aponta que as inter-relações horizontais são consideradas complexas, pela razão de que, demandam a capacidade empresarial e criativa da infraestrutura de C\&T e a estrutura produtiva. "Quando tais capacidades são inexistentes em um ou outro vértice - como os autores apontavam ser o caso da América Latina - o caminho para interrelações horizontais fica bloqueado.” Para resolver essa complexidade Sábato e Botana (1968) abordam que o instrumento mais adequado para facilitação do caminho entre as inter-relações horizontais seria a mobilidade de recursos humanos qualificados.

\subsection{Políticas públicas e o Estado}

No contexto analisado no Triângulo de Sábato, o governo tem o papel de desenvolver um ambiente favorável à inovação. O Estado precisa assumir o papel de articulador dos agentes envolvidos no processo de interação em prol da inovação. Para isto, é preciso que as políticas públicas destinadas a CT\&I sejam planejadas e formuladas de forma consistente para adequar às especificidades da sociedade aos objetivos legítimos do seu desenvolvimento.

Cassiolato e Lastres (2005 p. 8) tem a concepção de que o Estado é responsável pelos papéis de magnitude "seja como agente estruturante das forças produtivas seja como propulsor e orientador da sua difusão através da economia e sociedade".

Nesta conjuntura, a política pública tem um papel significativo em todos os setores de uma economia, visto que, através dela o Estado implanta o projeto governamental com ações voltadas para determinados setores da sociedade, visando trazer melhoras e resultados 
positivos. Para que o projeto possa ser realizado, envolvem uma diversidade de órgãos públicos, sistemas organizacionais e agentes relacionados.

As políticas públicas são aqui compreendidas como as de responsabilidade do Estado, já o monitoramento e manutenção, precisa de um processo de tomada de decisões que envolvem órgãos públicos, instituições e agentes da sociedade relacionados à política implementada. A política pública dedica-se no método de construir, executar decisões políticas e enfrentar o problema público (HOFLING, 2001; SECCHI, 2013).

Mesmo com um ambiente de complexidade, incerteza e riscos, é essencial investir em inovação e potencializar a capacidade tecnológica. O Estado é o articulador neste processo inovativo, sendo capaz de induzir fortemente, o comportamento, as estratégias e as decisões empresariais relativas à inovação. É de interesse do Estado desenvolver um arcabouço que permita a criação de novos conhecimentos e novas tecnologias com um ambiente competitivo e capaz de inovar (AVELLAR, 2007; SALERNO; KUBOTA, 2009; NASCIMENTO; LABIAK JUNIOR, 2011).

Nesta conjuntura, o Estado tem a responsabilidade de criar políticas tecnológicas com uma institucionalização forte e diversificada, para promover uma interação entre empresas, instituições de pesquisa e universidades. Para Salerno e Kubota (2008, p. 17-18) "Uma política de inovação parte da premissa de que o conhecimento tem, em todas as formas, um papel crucial no progresso econômico e é um fenômeno complexo e sistêmico", por isto, não é suficiente ter apenas a ciência, é primordial uma estrutura produtiva bem consolidada, e quem tem a capacidade de desenvolver isto, é o Estado. "Pela ação das instituições, o Estado, além de participar das interações, atua também como catalisador dos fluxos de conhecimento" (NASCIMENTO, 2011, p. 13).

Sabe-se que o motor da inovação está no acúmulo de aprendizado e fluxos de conhecimento, trazendo a necessidade do investimento tanto em capital físico quanto humano. Este investimento só será transformado em inovação se houver uma base institucional adequada e consolidada para CT\&I.

Dosi (1988) expõe três responsabilidades do Estado para atingir uma estrutura institucional adequada para o processo inovativo. Primeiramente o Estado como articulador, é necessário o alinhamento do interesse público com o privado. A segunda responsabilidade está na determinação do financiamento público, ou seja, onde ele será alocado para que resulte em desenvolvimento tecnológico. Por último, o Estado tem a responsabilidade de zelar pela propriedade intelectual. Com esse conjunto de ações é possível que o Estado alcance uma política industrial e de inovação eficiente. 
É considerada uma política de industrial e de inovação eficiente, se houver na conjuntura econômica do país, uma conexão entre ciência e tecnologia, criação de instituições consistentes para repassar os fluxos de conhecimento e acompanhar as transfigurações dos agentes ao longo do tempo. Ou seja, é indispensável que a política esteja bem definida para adequações de novas realidades e desafios (DOSI, 2008; NELSON, 2008).

\section{Interação da Universidade-Empresa}

A interação entre universidade-empresa surge como instrumento para o desenvolvimento de capacidades, aprendizagem e aquisição de conhecimento e tecnologias. A interação segundo Marques; Freitas e Silva (2007, p. 114) "também dilui os riscos relacionados à incerteza da atividade para inovação.” Deste modo, além do benefício das empresas nas economias de escala, elas passam a ter acesso a novas habilidades e recursos, e assim aperfeiçoando a evolução do conhecimento científico para capacidade tecnológica.

Estudar sobre universidade-empresa é relevante por fortalecer os sistemas de inovação. Os estudos de universidade-empresa atualmente estão sendo deslocados dos países desenvolvidos para países em desenvolvimento. Isto acontece, porque, além da interação de universidade-empresa fortalecer os sistemas de inovação, a mesma contribui para o progresso técnico (MANSFIELD, 1991; NARIN; HAMILTON; OLIVASTRO, 1997; YUSUF; NABESHIMA, 2006).

Para Bonaccorsi e Piccaluga (1994), as empresas interagem com as universidades para adquirir acesso às fronteiras científicas e acesso ao conhecimento de professores, pesquisadores e técnicos. Além disso, para redução dos custos operacionais e do desenvolvimento da empresa. A transferência do conhecimento da universidade traz o fortalecimento de capacidades inovativas das empresas. Na visão de Gielfi (2017, p. 27) “em outras palavras, a principal motivação da firma para interagir é identificar e explorar oportunidades tecnológicas".

O condicionamento da interação entre universidade-empresa na abordagem de Cohen; Nelson e Walsh (2002) são características estruturais como: tamanho da firma, o ambiente que a firma está inserida, intensidade de investimentos em P\&D entre outros. Deste modo, grandes empresas têm maior propensão de interação com as universidades. Outro condicionamento seria a capacidade que a firma tem de absorver o conhecimento externo e a escolha de estratégias de busca de conhecimentos (COHEN; LEVINTHAL, 1990; LAURSEN; SALTER, 2004). 
A qualidade de pesquisa acadêmica se encaixa como condicionante de interação entre universidade-empresa, pela razão de que, sendo a pesquisa relevante, os empresários terão interesse em investir e aplicar a pesquisa na empresa. O perfil dos pesquisadores acadêmicos é considerado como um condicionamento, devido aos empresários ter o interesse de contratálos para desenvolvimento de setores de P\&D dentro da empresa (MANSFIELD, 1991; D’ESTE; PATEL, 2007).

A interação universidade-empresa tem conquistado relevância nos estudos de inovação, pela razão de que, as universidades são fontes de conhecimentos para o processo inovativo e sua relação com as empresas é primordial para a boa performance do sistema de inovação. Diante disso, a maior parte dos países fizeram alterações em suas legislações, apostando em políticas que incentivam a interação universidade-empresa visando o desenvolvimento econômico tendo como suporte a pesquisa acadêmica. (LUNDVALL, 1992; ROSEMBERG; NELSON, 1994; GULBRANDSEN; MOWERY; FELDMAN, 2011).

\section{- Instituições científicas e tecnológicas (ICTs)}

Ao investigar uma conexão mais próxima entre a estrutura institucional e os processos de inovação é primordial analisar as funções básicas das instituições e seu papel específico em relação às inovações. Neste âmbito, as funções básicas das instituições são: i) redução da incerteza fornecendo informações; ii) regular conflitos; iii) administrar a cooperação; e, iv) prover incentivos.

Na primeira função, as instituições são fundamentais para que as organizações possam arriscar em diversos níveis de incerteza no processo inovativo, fornecendo informações para minimizar as dúvidas em relação ao comportamento dos agentes. A segunda e terceira função apresenta que as instituições têm o papel de regular os conflitos e administrar a cooperação. No processo inovativo existem conflitos (reestruturação organizacional, perda de poder, contratos, entre outros) que se sujeitam a normas e práticas jurídicas para adequação, logo é preciso uma administração para cooperação da estrutura institucional visando minimizar conflitos e incertezas. A última função refere-se a estimular o processo inovativo. O principal incentivo seria a interação entre firmas, universidades, governo (EDQUIST, 1997).

As instituições científicas e tecnológicas (ICTs) minimizam conflitos ligados aos processos inovativos e estimulam a interação e fluxo de conhecimento entre agentes: universidades, empresas e governo. São caracterizadas como órgão ou entidade da administração pública com o propósito de executar atividades de pesquisa científica e 
tecnológica e como suporte para transferência de conhecimento, nascimento e desenvolvimento de empresas. As instituições científicas e tecnológicas (ICTs) são constituídas por: incubadoras, cidades inovadoras, núcleos de inovação tecnológica, redes de inovação, plataformas tecnológicas, entre outras (BRESCHI; MALERBA, 1997; RUSSO et. al, 2012; CASSIOLATO; SZAPIRO, 2015).

A ideia de ICTs teve início na região do Vale do Silício em 1937. A universidade de Stanford criou a Stanford Research Park, que era considerado um parque tecnológico responsável por transferir tecnologia da universidade para as empresas. Após isso, houve a criação de várias outras incubadoras nos Estados Unidos, e em sequência propagou-se para Europa (WOLFFENBÜTEL, 2001).

No Brasil, as incubadoras surgiram apenas na década de 80. Em 1982, o Conselho Nacional de Desenvolvimento Científico e Tecnológico (CNPq) juntamente com o governo instituiu o Programa de Tecnologia e Inovação. Este programa tinha o propósito de incentivar a interação entre universidades e empresas, por meio deste programa, surgiram as incubadoras tecnológicas. A primeira delas foi a São Carlos (SP) em 1984, considerada a mais antiga da América Latina. Em 1897 com a criação da Associação Nacional de Entidades Promotoras de Empreendimentos Inovadores (ANPROTEC) houve a aproximação das incubadoras nas empresas, facilitando assim, a transferência de conhecimento (WOLFFENBÜTEL, 2001; ANPROTEC, 2009).

Em 2005 o Ministério de Ciência e Tecnologia (MCT) criou o Programa Nacional de Apoio às Incubadoras de Empresas e Parques Tecnológicos (PNI), este programa existe até hoje com o intuito de dar suporte as incubadoras visando ampliar e otimizar recursos para serem conduzidos no apoio, geração e consolidação de empresas inovadoras (MCT, 2009).

No contexto de ICTs, Mansano e Pereira (2015) afirmam que as incubadoras se destacam no sistema de inovação do Brasil e, são caracterizadas como entidades promotoras de empreendimentos inovadores. Uma incubadora de empresas realiza o processo de geração e consolidação de micro e pequenas empresas, facilitando o desenvolvimento da empresa até chegar à inovação tecnológica. Deste modo, têm como propósito estimular a criação e ou o desenvolvimento de micro e pequenas empresas, e com isso fornecer capacitação para que possam atingir autonomia tecnológica e contribuir para o desenvolvimento do sistema produtivo nacional.

A incubadora é um arranjo institucional estruturado para estimular e facilitar a vinculação empresa e universidade, fortalecendo a interação entre universidades e empresas, além de atrelar o setor produtivo com instituições de apoio, ensino e pesquisa, financiamentos 
governamentais entre outros. Neste sentido, há consenso de que a inovação não surge de forma espontânea, é preciso que haja investimento em pesquisa e desenvolvimento. É preciso criar instrumentos e políticas, canalizando esforços e recursos com o objetivo primordial de gerar e expandir empresas, criar e aperfeiçoar uma inovação, transferir conhecimento em CT\&I para poder contribuir para desenvolvimento econômico sustentado e competitivo.

Uma incubadora é um arranjo interinstitucional com a função de intensificar e facilitar a relação entre universidade, setor produtivo e inúmeras instituições de apoio. As Incubadoras de Ciência e Tecnologia, são voltadas para atividades de pesquisa, desenvolvimento e inovação que envolvam risco tecnológico, para resolver o problema técnico específico ou obtenção de produto, serviço ou processo inovador. Outra função seria que a incubadora oferece o suporte não apenas na transferência de conhecimento, mas, em fornecer instalações físicas, serviços de apoio, assessorias, consultorias entre outros. Neste sentido, as incubadoras tecnológicas contribuem para SNI por serem instrumentos de catalisação do conhecimento gerado nas universidades e centros de pesquisas (VEDOVELLO; PUGA; SINTPq, 2013).

Existem algumas exigências por parte das incubadoras para que a empresa possa solicitar o auxílio: i) processo de seleção; ii) processo de pré-incubação; e, iii) processo de incubação. No processo de seleção, a maioria das incubadoras realiza uma análise de viabilidade do projeto da empresa, semelhanças do projeto da empresa com os objetivos da incubadora, comprovações de recursos financeiros para sustentação da fase inicial até a estabilização da empresa. Neste processo, busca identificação das propostas do projeto que possuem maior perspectiva de sucesso (IDISC, 2009).

O processo de pré-incubação tem a responsabilidade de amparar os empreendedores nos estágios iniciais de abertura da empresa, através de consultorias, cursos, eventos, treinamentos e interação da universidade com os centros de pesquisa e a empresa. Outra função do processo de pré-incubação é a redução de riscos que a maioria das empresas passam nos primeiros anos. Após a empresa ter passado pela pré- incubação e atingindo os objetivos de formalização jurídica, do plano de negócios e do produto/serviço, a empresa está apta para o processo de incubação. Neste processo, o principal objetivo é a consolidação no mercado. Atingindo este objetivo a empresa pode implantar estratégias de desenvolvimento e crescimento, mas, para isso é preciso identificar as necessidades da empresa para crescer e desenvolver juntamente com as forças do mercado (FELIX, 2001; IDISC, 2009).

No processo de incubação ocorre o fortalecimento da interação universidade, empresa incubada e instituição de pesquisa através da transferência de tecnologia da universidade para a empresa de maneira mais definida. Maior parte das incubadoras encaminha os 
empreendedores para os Núcleos de Inovação Tecnológica para formalização de contratos de transferência, cooperação e patentes. O período de incubação tem a duração de 3 anos e o de pré-incubação aproximadamente 1 ano. Após este período a empresa já consolidada precisa montar seu espaço independente e deixar a incubadora (IDISC, 2009).

A lei de Inovação Tecnológica $\left(n^{\circ} 10.973 / 2004\right)$ trouxe um grande desafio para as ICTs do país: a expansão e fortalecimento dos Núcleos de Inovação Tecnológica (NITs). Neste sentido, os NITs tornaram-se protagonistas do processo de interação universidadeempresa e passou a ter uma intensa participação no processo de inovação.

O NIT tem a finalidade de garantir a gestão da política institucional de inovação. Os NITs foram criados para dar suporte nos aspectos legais, financeiros, técnicos e de marketings para pesquisadores universitários e empresas no processo de transferência de tecnologia. Geralmente fazem parte da estrutura administrativa central da universidade (LIPINSKI; MINUTOLO; CROTHERS, 2008; TORKOMIAN, 2009). Benedetti (2010, p. 7) afirma que o NIT é um mediador que atua como "interlocutor entre a universidade e a empresa, os pesquisadores participam apenas quando são imprescindíveis e não se envolvem na discussão jurídica e comercial, concentrando-se em sua principal competência que é a pesquisa científica e o ensino".

Nesse processo de ICT-empresa, os NITs têm o objetivo de criar um ambiente colaborativo que favoreça e ampare os pesquisadores nas questões de propriedade intelectual, e direcione as questões à Agência de Inovação, que delibera sobre quais processos e produtos gerados pela atividade de pesquisa devem ser objeto de pedido de proteção.

Na figura 4 é apresentada a função do Núcleo de Inovação Tecnológica (NIT). O governo como principal agente se enquadrando em dar suporte para as Universidades, incubadoras, NIT e empresas no desenvolvimento de políticas públicas, fomento e subsídios para CT\&I. O NIT localizado no centro das universidades, incubadoras e empresas, atuando como o agente facilitador da relação entre universidade e o setor produtivo por meio das incubadoras e parques tecnológicos.

Figura 2 - Função do Núcleo de Inovação Tecnológica (NIT) 


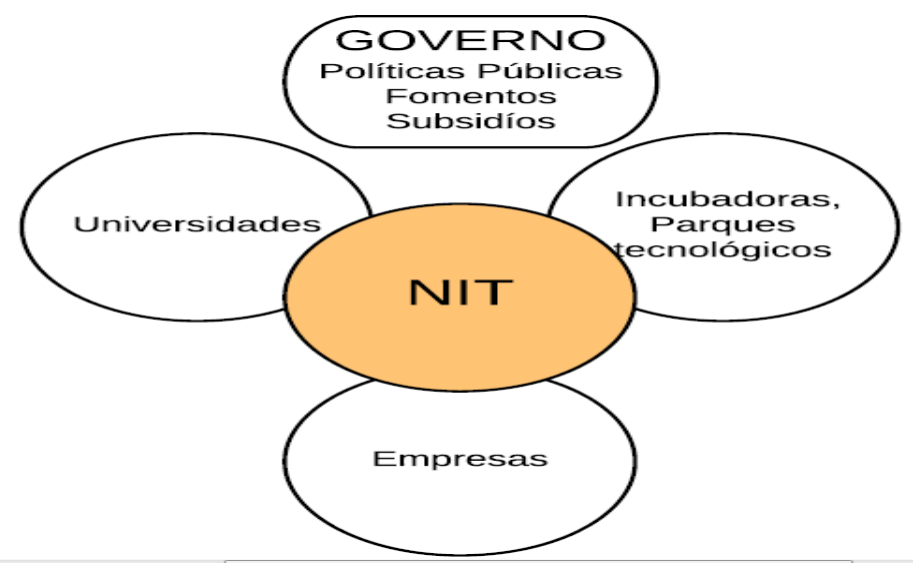

Fonte: Elaboração própria. FORTEC, 2017.

No quadro 2 é definido as competências mínimas do Núcleo de Inovação Tecnológica (NIT) dentro da universidade. Nessas competências estão: zelo pela política institucional, avaliação dos resultados, desenvolvimento e acompanhamento do relacionamento das instituições científicas e tecnológicas com as empresas.

Quadro 2 - Competências mínimas do Núcleo de Inovação Tecnológica (NIT)

\begin{tabular}{|c|}
\hline Competências mínimas do Núcleo de Inovação Tecnológica (NIT) \\
\hline $\begin{array}{c}\text { I - zelar pela manutenção da política institucional de estímulo à proteção das } \\
\text { criações, licenciamento, inovação e outras formas de transferência de } \\
\text { tecnologia; }\end{array}$ \\
II - avaliar e classificar os resultados decorrentes de atividades e projetos de \\
pesquisa;
\end{tabular}

Fonte: Elaboração própria. BENEDETTI, 2010; BRASIL, 2016; MACHADO; SARTORI; CRUBELLATE, 2017. 
Dias (2010) realizou uma pesquisa de 157 NITs brasileiros e afirmou que a expansão dos NITS foi concentrada no ano de 2004, ou seja, ano da criação da Lei da Inovação. A situação atual dos NITs no Brasil tem seu registro por dados coletados pelo Formict e analisados pelo MCTI. Em 2014 esse formulário foi respondido por 264 Instituições, sendo 194 públicas e 70 privadas.

A pesquisa revelou que no Brasil existem 180 instituições, sendo que os NITs implementados são apenas 68,2\%, fase de implementação (20,5\%) e 11,4\% dos NITs ainda passam por processo de análise antes de entrar na fase de implementação. Os dados dos relatórios posteriores mostram o crescimento da formalização e do número de NITs. Neste sentido, um dos desafios da Lei Novo Marco legal (CT\&I) no Brasil (Lei nº 13.243/2016) é alcançar a totalidade da implementação dos NITs nas instituições públicas e estabelecer aqueles já implementados (MCTI, 2015).

\section{- Dados das instituições científicas e tecnológicas no Brasil}

Desde a implantação da Lei da inovação todas as ICTs são obrigadas a preencher o Formulário para Informações sobre a Política de Propriedade Intelectual, Científicas, Tecnológicas e de Inovação (FORMICT). Este formulário foi elaborado pelo Ministério de Ciência, Tecnologia e Inovação (MCTI) com intuito de acompanhar o desenvolvimento da propriedade intelectual e das ICTs. Através deste relatório existe a possibilidade de avaliar, diagnosticar e planejar políticas públicas para inovação tecnológica e para consolidar a transferência de tecnologia entre universidade-empresa (BRASIL, 2013).

Os dados desta seção foram retirados com base no relatório publicado pelo MCTI: Política de Propriedade Intelectual das Instituiçoes Científicas e Tecnológicas do Brasil (Relatório FORMICT - 2017). Na Tabela 2 - Quantidade de ICTs no Brasil até 2016 percebese um pequeno crescimento das instituições científicas de tecnologias que responderam o formulário. Em 2014 eram 264 instituições e em 2017 o total de 278 ICTs (193 públicas e 85 privadas).

Tabela 1 - Quantidade de ICTs no Brasil até 2017

\begin{tabular}{|l|c|c|}
\hline Natureza da Instituição & Quantidade & $\%$ \\
\hline Privada & 85 & 30,6 \\
\hline Pública & 193 & 69,4 \\
\hline
\end{tabular}


Fonte: BRASIL, 2017.

Os Núcleos de Inovação Tecnológica (NITs) considerado como o órgão com a função de gestão dos contratos de transferência de tecnologia das ICTs para as empresas vem crescendo desde 2010. A figura 4 - Evolução das implementações dos NITs mostra um aumento significativo de implementações nos últimos anos, há ainda 46 NITs que estão em fase de implementação e 24 não foram implementados.

Gráfico 1 - Evolução das implementações dos NITs

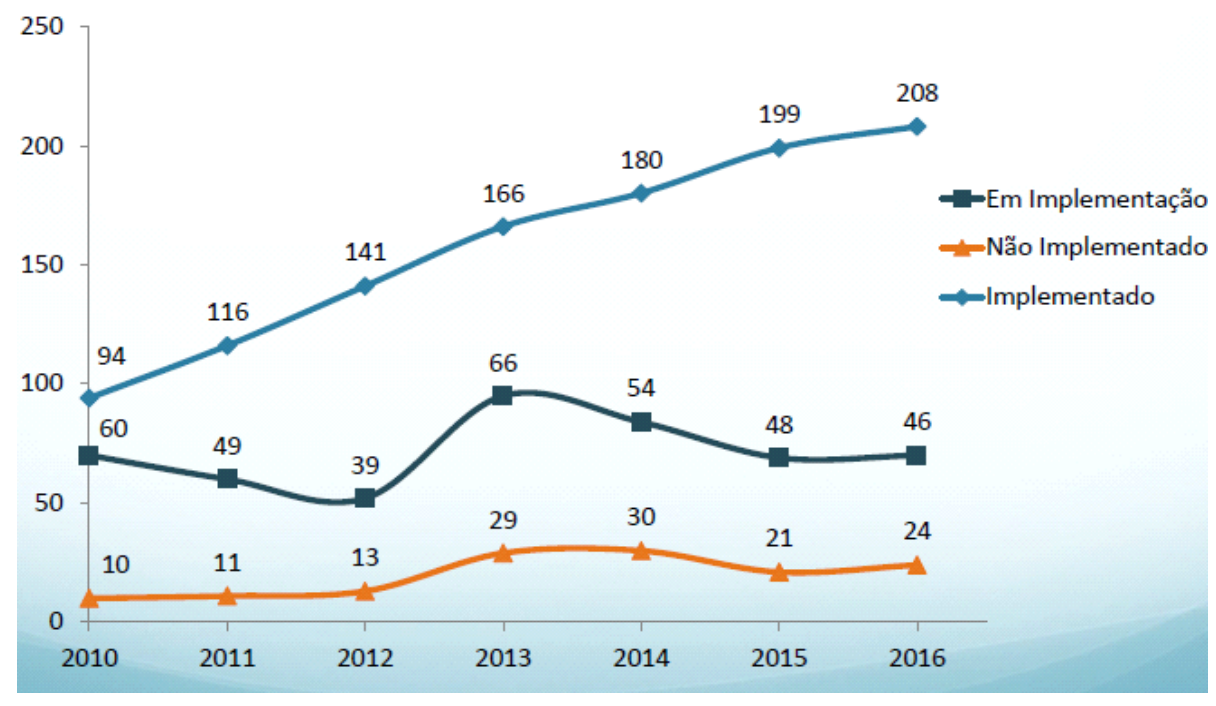

Fonte: BRASIL, 2017.

Na tabela 1, apresenta os rendimentos recebidos com os contratos de Tecnologia, foram analisados contratos firmados no ano base de 2016 ou que tenham sido firmados em anos anteriores, mas geraram recursos no ano de 2016. As ICTs públicas contabilizaram R\$101,4 milhões de rendimentos no ano de 2016 relacionado à Transferência de Tecnologia. As ICTs privadas contabilizaram R $\$ 124,9$ milhões de rendimentos. Referente 
ARTIGO

: INOVAÇÃO

aos gastos com registros e manutenção da Política da Propriedade Intelectual no ano de 2016, as ICTs públicas contabilizaram $\mathrm{R} \$ 7,9$ milhões e as privadas $\mathrm{R} \$ 1,3$ milhões.

Tabela 2 - Rendimentos dos contratos e gastos com propriedade intelectual em 2016

\begin{tabular}{|l|c|c|}
\hline Recursos & Valores $(\mathbf{R} \$)$ & Valores $(\mathbf{R} \$)$ \\
\hline $\begin{array}{l}\text { Rendimentos no ano percebidos na transferência de } \\
\text { tecnologia }\end{array}$ & $\begin{array}{c}\text { Pública } \\
\text { Privada }\end{array}$ \\
\hline \begin{tabular}{l} 
Gastos com registros e manutenção da PI \\
\hline
\end{tabular} & $7.938 .529 .554,08$ & $124.941 .068,65$ \\
\hline
\end{tabular}

Fonte: BRASIL, 2017.

No gráfico 2 - Montante de contratos de tecnologia firmados em 2016, alcançou-se o valor total de $\mathrm{R} \$ 437,9$ milhões de contratos de tecnologia firmados, prevalecendo a realização de contratos Sem Exclusividade totalizando nas ICTs privadas R \$1 milhão e nas públicas R \$199,3 milhões. Com relação aos contratos Com Exclusividade, atingiu-se R \$77,2 milhões nas privadas e $\mathrm{R} \$ 38,5$ de Contratos de Tecnologia nas ICTs públicas. Nos contratos de Outras Formas foram contabilizados $\mathrm{R} \$ 6,2$ milhões na privada e $\mathrm{R} \$ 115,5$ na pública. Na contagem geral do valor total de $\mathrm{R} \$ 437,9$ milhões de contratos firmados de tecnologia, sendo que 45,51\% desses recursos correspondem a contratos de ICTs públicas sem exclusividade, 26,37\% de contratos de Outras Formas de ICTs Públicas e 17,62\% de contratos de tecnologia Com Exclusividade por ICTs privadas.

Gráfico 2 - Montante dos contratos de tecnologia firmados em 2016

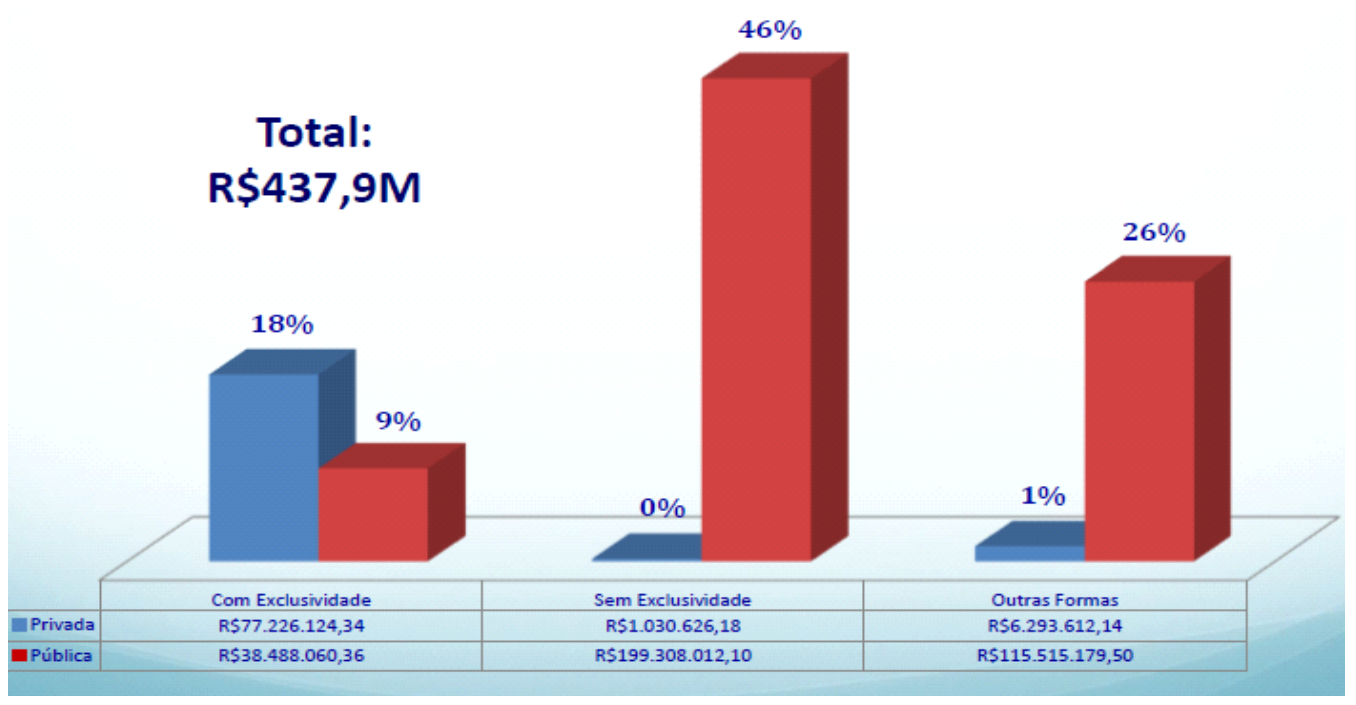

Fonte: BRASIL, 2017. 
Figura 3 - Atividades essenciais do NIT

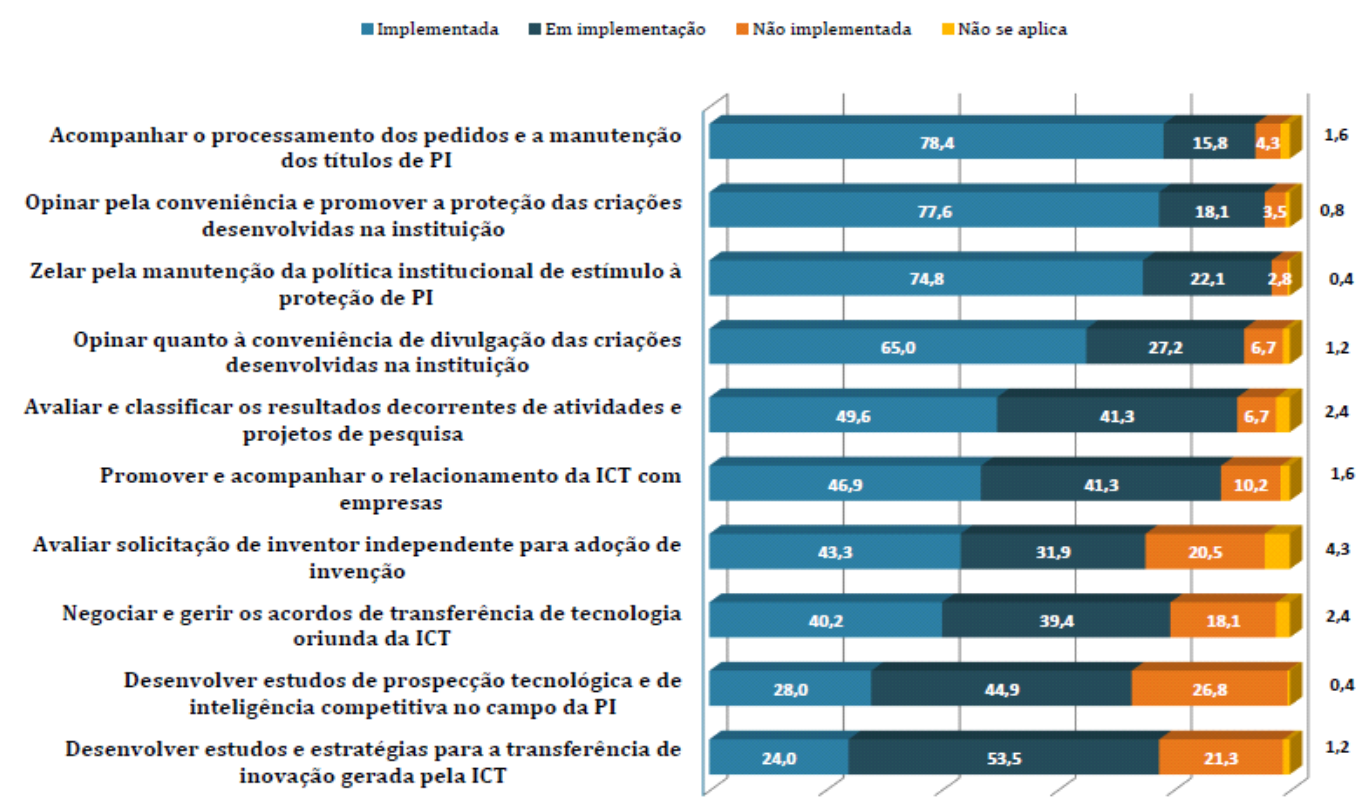

Fonte: BRASIL, 2017.

$\mathrm{Na}$ figura 5 - Atividades essenciais do NIT, que seriam as competências mínimas abordadas no quadro 2 , destaca-se que $78,4 \%$ da atividade de acompanhar o processamento dos pedidos e a manutenção dos títulos de Propriedade Intelectual (PI) foi implementada. A atividade de opinar pela conveniência e promover a proteção das criações desenvolvidas na instituição foi $77,6 \%$ implementada. No que se refere ao zelo da política institucional de estímulo à proteção PI 74,8\% da atividade foi implementada. Na atividade considerada uma das mais importantes para fortalecimento da relação universidade-empresa: Desenvolver estudos e estratégias para a transferência de inovação gerada pela ICT é implementado apenas $24 \%$ da atividade, enquanto 53,5\% ainda está em fase de implementação e 21,3\% não implementada.

\section{CONCLUSÃO}

Percebe-se o quanto é primordial a interação entre Universidade, Empresa e Estado na dinâmica do sistema capitalista. O Estado teria o importante papel de ser o articulador neste processo inovativo, sendo capaz de induzir fortemente o comportamento, as estratégias e as decisões empresariais. As instituições têm o relevante papel de fortalecer a transferência de conhecimento entre universidade-empresa no desenvolvimento de capacidades, aprendizagem e aquisição de conhecimento e tecnologia. 
No Brasil a política de inovação de 2004 teve o desafio de expandir as instituições científicas e tecnológicas, porém ainda existe um arcabouço burocrático a ser resolvido. Existem no total 264 instituições científicas e tecnológicas, mas apenas $68 \%$ delas estão implementadas. Como analisado houve um progresso em contratos de tecnologia, criação e implementação dos núcleos de inovação tecnológica e incubadoras, portanto, tem-se a necessidade de facilitar a interação da universidade-empresa, para que as instituições científicas e tecnológicas continuem expandindo. Com este intuito, o sancionamento em 2016 da política Novo Marco Legal (CT\&I) está em ação buscando a facilitação da transferência de conhecimento e a interação entre Universidade-Empresa-Estado por meio da redução de aspectos burocráticos. 


\section{REFERENCIAS}

ABRAMOVITZ, M. Resource and output in the United States since 1870. In: ROSENBERG, N. (org.). The Economics of Technological Change. Londres: Penguin Books, 1971.

ALBUQUERQUE, E. M. Produção científica e sistema nacional de inovação. Ensaios FEE, v. 1, n.1, p. 156-180, 1998.

ALBUQUERQUE, E. M. Sistema Nacional de Inovação no Brasil: uma análise introdutória partir de dados disponíveis sobre a ciência e a tecnologia. Revista de Economia Política, v.16, n. 3, p. 56-72, jul/set, 1996.

ALBUQUERQUE, E. M; SICSÚ, J. Inovação Institucional e Estímulo ao Investimento Privado. São Paulo em Perspectiva, v. 14, n. 3, jul./set. 2000. Disponível em: http://www.scielo.br/pdf/spp/v14n3/9778.pdf. Acesso em: 12 out. 2018.

AVELLAR, A. P. M. Avaliação de políticas de fomento à inovação no Brasil: impacto dos incentivos fiscais e financeiros em 2003. 2007. Tese (Doutorado em Economia) - Instituto de Economia Universidade Federal do Rio de Janeiro, Rio de Janeiro, 2007.

AVELLAR, A. P. M. Avaliação do impacto do PDTI sobre o gasto em atividades de inovação e P\&D das empresas industriais. In: DE NEGRI, J. A.; KUBOTA, L. C. Políticas de Incentivo à Inovação Tecnológica no Brasil. Brasília: IPEA, 2008. p. 323-358. Cap. 8.

AVELLAR, A.; OLIVEIRA, F. Comportamento do Sistema Nacional de Inovação Brasileiro (2000-2007). Revista Economia Ensaios, v. 23, n. 1, 2008. Disponível em: http://www.seer.ufu.br/index.php/revistaeconomiaensaios/article/view/2030. Acesso em: 02 set. 2018.

BONACORSI, A.; PICCALUGA, A. Atheorical framework for the evaluation of universityindustry relationships. R\&D Management, v. 3, n. 24, p. 229-247, 1994.

BRASIL. Ministério da Ciência, Tecnologia e inovação. Política de Propriedade Intelectual das Instituições Científicas e Tecnológicas do Brasil: Relatório Formict 2014. Brasília, 2015.

BRASIL. Ministério da Ciência, Tecnologia e Inovação. Política de propriedade intelectual das instituições científicas e tecnológicas do Brasil: relatório FORMICT 2017. Brasília, 2017.

BRESCHI, S.; MALERBA, F. Sectoral innovation systems: technologica regimes, schumpeterian dynamics, and spatial boundaries. In: EDQUIST, C. (ed.). Systems of innovation: technologies, institutions and organizations. London: Pinter, 1997. p. 130-156.

CASSIOLATO, J. E.; LASTRES, H. M. Sistemas de Inovação: políticas e perspectivas. São Paulo em Perspectiva, v. 19, n. 1, 2005. Disponível em: http://www.scielo.br/pdf/spp/v19n1/v19n1a03.pdf. Acesso em: 15 out. 2018. 


\section{INOVAÇÃO}

CASSIOLATO, J. SZAPIRO, M. Os dilemas da política industrial e de inovação: os problemas da Região Sudeste são os do Brasil. In: Leal, C. F. et al. (org). Um olhar territorial para o desenvolvimento Sudeste. Rio de Janeiro, 2015. Produção Banco Nacional de Desenvolvimento Econômico e Social (BNDES),

COHEN, W. M.; LEVINTHAL, D. A. Absorptive capacity: a new perspective on learning and Innovation. Administrative Science Quarterly, v. 35, n. 1, p. 128, mar. 1990.

D'ESTE, P.; PATEL, P. University-industry linkages in the UK: What are the factors underlying the variety of interactions with industry? Research Policy, v. 36, n. 9, p. 12951313, nov. 2007.

DIAS, J. C. Competências Organizacionais diferenciadoras em núcleos de inovação tecnológica. 2010. 209 f. Dissertação (Mestrado em Administração) - Universidade Federal do Paraná, Curitiba, 2010.

DOSI, G. Sources, procedures and microeconomic effects of innovation. Journal of Economic Literature, v. 26, n. 3, p. 1120-1171. 1988.

EDQUIST, C. The systems of innovation approach and innovation policy: an account of the state of art. DRUID Conference, 2001.

FAGERBERG, J.; SRHOLEC, M.; KNELL, M. The Competitiveness of Nations: Why Some Countries Prosper While Others Fall Behind World Development, v. 35, n. 10, 1595-1620, 2007.

FREEMAN, C. Technology policy and economic performance: lessons from Japan. London: Pinter, 1987.

FREEMAN, C.; SOETE, L. A economia da inovação industrial. Campinas: Editora UNICAMP, 2008.

FRENKEL, A. "Why high-technology firms choose to locate in or near metropolitan areas". Thousand Oaks: Urban Studies, v. 38, 7, p. 1083-1101, 2001.

FORTEC. Fórum Nacional de Gestores de Inovação e transferência de tecnologia. Presidência, Diretoria e Diretório. Material de Palestra. In: Encontro Acadêmico de Propriedade Intelectual, Inovação e Desenvolvimento (ENAPID), 10., 2017. Rio de Janeiro. 2017.

FURTADO, C. Desenvolvimento e Subdesenvolvimento. Rio de Janeiro: Editora Fundo Cultura, 1961.

GIELFI, G. A interação Universidade-Empresa na Indústria de petróleo brasileira. 2017. Tese (Doutorado em Geociências) - Universidade Estadual de Campinas, Campinas, 2017.

GULBRANDSEN, M.; MOWERY, D.; FELDMAN, M. Introduction to the special section: heterogeneity and university-industry relations. Research Policy, v. 40, p.1-5, 2011. 
HOFLING, E. Estados e políticas (públicas) sociais. Cadernos Cedes, ano XXI, n. 55, p. 3041, nov. 2001.

IDISC. InfoDev Incubator Support Center. 2009. Disponível em: http://www.idisc.net. Acesso em: 25 set. 2018.

JUNG, J.; MAH, J. R. D policies of Korea and their implications for developing countries. Science. Technology and Society, v. 18, n. 2, p. 165-188, 2013.

LAURSEN, K.; SALTER, A. Searching high and low: what types of firms use universities as a source of innovation? Research Policy, v. 33, n. 8, p. 1201-1215, 2004.

LIPINSKI, J.; MINUTOLO, M. C.; CROTHERS, L. M. The complex relationship driving technology transfer: the potential opportunities missed by universities. Institute of Behavioral and Applied Management, v. 9, n. 2, p. 112-133. Jan. 2008.

LUNDVALL, B. (ed.). National systems of innovation: towards a theory of innovation and interactive learning. London: Pinter, 1992a.

LUNDVALL, B. (ed.). Introduction. In: LUNDVALL, B. (Ed.). National systems of innovation: towards a theory of innovation and interactive learning. London: Pinter, 1992b, p. 1-19.

LUNDVALL, B. (ed.). National innovation systems: analytical concept and development tool.

Industry and Innovation, v. 14, n. 1, p. 95-119. 2004.

MACALFE, S. Technology systems and technology policy in an evolutionary framework. Cambridge Journal of Economics, v. 19, n. 1, p. 25-46, 1995.

MANSFIELD, E. Academic Research and Industrial Innovation. Research Policy, v. 20, n. 1, p. 1-12, 1991.

MANSANO, F H; PEREIRA, M F. Incubadoras de Empresas como mecanismos de apoio ao desenvolvimento econômico. In: CONFERENCIA ANPROTEC DE EMPREENDEDORISMO E AMBIENTES DE INOVAÇÃO, 25. 2015. Anais eletrônicos. Cuiabá-MT. 2015.

NASCIMENTO, D. E.; LABIAK JUNIOR, S. Ambientes e dinâmicas de cooperação para inovação. Curitiba: Aymará, 2011.

NELSON, R.; ROSENBERG, N. National innovation systems: a comparative analysis. New York: Oxford University Press, 1993.

NELSON, R.; WINTER, S. An evolutionary theory of economic change. Cambridge: Harvard University, 1982.

NELSON, R. R. Economic development from the perspective of evolutionary economic theory. Oxford Development Studies, v 36 n. 1, p. 10-21, 2008. 


\section{INOVAÇÃO}

NONAKA, I.; VON KROGH, G.; VOELPEl, S. Organizational knowledge creation theory: Evolutionary paths and future advances. Organization Studies, v. 27, n. 8, p. 1179-1208, 2006.

ORGANISATION FOR ECONOMIC CO-OPERATION AND DEVELOPMENT. National Innovation Systems. Paris: OECD, 1997.

PATEL, P.; PAVITT, K. National Innovation Systems: why they are important, and how they might be measured and compared. Economics of Innovation and New Technology, v. 3, n. 1, p. 77-95, 1994.

ROSENBERG, N.; NELSON, R. R. American universities and technical advance in industry. Research Policy, v. 23, n. 3, p. 323-348, maio 1994.

RUSSO, S. L. et al. Propriedade intelectual. RUSSO, S. L.; SILVA, G. F.; NUNES, M. A. S. N. (org.). In: RUSSO, S. L. et al. Capacitação em inovação tecnológica para empresários. São Cristóvão: Editora UFS, 2012. p. 55-90.

SÁBATO, J.; BOTANA, N. La ciencia y la tecnología en el desarrollo futuro de América Latina. Revista de la Integración, v. 3, p. 15-36, 1968.

SALERNO, M.; KUBOTA, L. Estado e inovação. In: NEGRI, J. KUBOTA, L. (org). Políticas de Incentivo à Inovação Tecnológica. Brasília: Instituto de Pesquisa Aplicada (IPEA), 2008. cap. 1.

SBICCA, A.; PELAEZ, V. Sistemas de Inovação. In: PELAEZ, V.; SZMERECSÁNYI, T. (org). Economia da Inovação Tecnológica. São Paulo: Editora Hucitec, 2006.

SECCHI, L. Políticas Públicas: conceitos, esquemas de análise, casos práticos. Stamford, Connecticut: Cengage Learning. 2013.

SINDICATO DOS TRABALHADORES EM PESQUISA, CIÊNCIA E TECNOLOGIA (SINTPq). Os desafios dos núcleos de inovação tecnológica (NITs) no Brasil. São Paulo-SP. 2014. Disponível em: http://sintpq.org.br/index.php/blog/item/3312-os-desafios-dos-nucleosde-inovacao-tecnologica-nits-no-brasil. São Paulo-SP. 2014. Acesso em: 02 de jul de 2018.

STRACHMAN, E.; DEUS, A. Instituições, inovações e sistemas de inovação: interações e precisão de conceitos. Ensaios FEE, v. 26, n. 1, p. 575-604. Jun. 2005.

SUZIGAN, W.; ALBUQUERQUE, E. M. A interação entre universidades e empresas em perspectiva histórica no Brasil. Texto para Discussão, n. 329, 2008.

TORKOMIAN, A. L. V. Panorama dos núcleos de inovação tecnológica no Brasil. In: TORKOMIAN, A. L. V. et al. Transferência de tecnologia: estratégias para estruturação e gestão de núcleos de inovação tecnológica. Campinas, SP: Komedi, 2009.

VEDOVELLO, C.; PUGA, F. P.; FELIX, M. Criação de Infra-Estruturas Tecnológicas: A Experiência Brasileira de Incubadoras de Empresas. Revista do BNDES, Rio de Janeiro, v. 8, n. 16, p. 183-214, dez., 2001. 
WOLFFENBÜTTEL, A. Avaliação do Processo de Interação Universidade-Empresa em Incubadoras Universitárias de Empresas: um Estudo de Caso na Incubadora de Empresas de Base Tecnológica da UNISINOS. 2001. Dissertação (Mestrado em Administração) - UFRS, Rio Grande do Sul, 2001.

YUSUF, S.; NABESHIMA, K. Postindustrial east Asian cities: innovation for growth. Washington, DC: World Bank and Stanford University Press, 2006. 